\title{
Treatment of Inpatient Asymptomatic Hypertension: Not a Call to Act but to Think
}

\author{
James Anstey, MD ${ }^{1 *}$; Brian P Lucas, MD, MS 23,4
}

${ }^{1}$ Division of Hospital Medicine, Department of Medicine, University of California, San Francisco, San Francisco, California; ${ }^{2}$ Medicine Service, White River Junction VA Medical Center, White River Junction, Vermont; ${ }^{3}$ Geisel School of Medicine at Dartmouth College, Hanover, New Hampshire; ${ }^{4}$ The Dartmouth Institute for Health Policy \& Clinical Practice, Hanover, New Hampshire.

Y

our pager beeps. Your patient, Mrs. Jones, who was admitted with cellulitis and is improving, now has a blood pressure of 188/103 on routine vitals. Her nurse reports that she is comfortable and asymptomatic, but she met the "call parameters." You review her chart and find that since admission her systolic blood pressure (SBP) has ranged from 149 to $157 \mathrm{~mm} \mathrm{Hg}$ and her diastolic blood pressure (DBP) from 84 to $96 \mathrm{~mm} \mathrm{Hg}$. Her nurse asks how you would like to treat her.

While over half of inpatients have at least one hypertensive episode during their stay, evidence suggests that nearly all such episodes-estimates are between 98\% and 99\%1,2-should be treated over several days with oral antihypertensives, not acutely with intravenous medications. ${ }^{3-6}$ Current guidelines recommend that intravenous medications should be reserved for severe hypertensive episodes (SBP > 180, DBP > 120) with acute end-organ damage, ${ }^{7,8}$ but such "hypertensive emergencies" are rare on the general medicine wards. Still, hospitalists regularly face the dilemma posed by Mrs. Jones, and evidence shows they often prescribe intravenous antihypertensives. . $^{1,5}$ This unnecessary use can lead to unreliable drops in blood pressure and exposes our patients to potential harm.,

In this issue of the Journal of Hospital Medicine, two papers describe the frequency of inappropriate intravenous antihypertensive use in their hospitals and the subsequent quality improvement efforts implemented to reduce this practice. The first, by Jacobs et al., found that over a 10-month period, 11\% of patients who experienced "asymptomatic hypertension" on an urban academic hospital medicine service were treated inappropriately with intravenous antihypertensives, ${ }^{9}$ with $14 \%$ of those experiencing an adverse event. The second paper, by Pasik et al., found that in their urban academic medical center there were 8.3 inappropriate intravenous antihypertensive orders placed per 1,000 patient days, ${ }^{10}$ with nearly half of those treated experiencing an adverse event. Based on these findings, each group then led interventions to reduce the use of intravenous antihypertensives. *Corresponding Author: James Anstey, MD; E-mail: james.anstey@ucsf.edu;
Telephone: 415 476-4997; Twitter: @jeanstey.

Received: December 14, 2018; Revised: December 30, 2018;

Accepted: January 2, 2019

C 2019 Society of Hospital Medicine DOI 10.12788/jhm.3160
While both groups engaged physicians and nurses as primary stakeholders, Pasik et al. ${ }^{10}$ worked to further expand nursing staff roles by empowering them to assess for underlying causes of hypertension, such as pain or anxiety, as well as end-organ damage via specific guided algorithms prior to contacting physicians. In doing so, they reduced intravenous antihypertensive use by $60 \%$ during the postintervention period, with a proportional reduction in adverse events. In addition to their educational initiative, Jacobs et al. aimed to limit calls by liberalizing the "ceiling" on standard nursing call parameters for blood pressure from 160/80 to 180/90. Following their intervention, intravenous antihypertensive orders were reduced by $40 \%$, with the mean orders per patient with asymptomatic hypertension decreasing from $11 \%$ to $7 \%$.

While these results are admirable, some caution in their interpretation is needed. For example, Jacobs et al. used electronic health record data to retrospectively identify hypertension as "symptomatic" or "asymptomatic" using laboratory, electrocardiogram, and imaging diagnostics as surrogate markers for "provider concern for end-organ damage." Although it appropriately focused on concern for end-organ damage as justification for intravenous antihypertensives, this approach potentially underappreciated true hypertensive emergencies, thereby overestimating the amount of inappropriate use of intravenous antihypertensives. Pasik et al. utilized chart review of patients prescribed intravenous antihypertensives and therefore did not explore how often symptomatic hypertension occurred in patients who did not receive intravenous antihypertensives. Subsequently, this limited their ability to evaluate unintended harms of their initiative. To address this limitation, the authors followed a group of 111 patients who had elevated hypertension but did not receive intravenous antihypertensives and found no adverse outcomes. ${ }^{10}$ Because both studies were retrospective in nature, they were subject to biases from providers choosing intravenous antihypertensives for reasons that were neither captured by their datasets nor adjusted for. Additionally, neither study reported downstream impacts such as an increase in symptomatic hypertensive episodes or more rare events such as kidney injury, stroke, or myocardial infarction.

Given that guidelines discourage using intravenous antihypertensives, why were the efforts of Jacobs et al. ${ }^{9}$ and Pasik et al. ${ }^{10}$ needed in the first place? In a recent installment of Choosing Wisely: Things We Do For No Reason, Breu et al. ${ }^{11}$ cite two primary reasons: first, providers have unfounded fears that asymptomatic hypertension will quickly progress to cause organ 
damage; second, providers lack understanding of the potential harms from overtreatment. It is fitting, therefore, that both groups of authors focused on these topics in their education initiatives for physicians and nurses. Yet, as good quality improvement requires steps beyond education, it was promising to see that both authors additionally focused on intervening to change the systems and culture that existed around physician and nursing communication.

In the age of electronic health records, there has been a sustained focus on creating standardized order sets. While the value of these order sets has been widely demonstrated, there are downsides. For example, nursing call parameters in admission order sets are rarely patient-specific but account for a significant portion of nursing and physician communication. These one-size-fits-all orders limit nurses from using their clinical training and create unnecessary tensions as nurses are obligated to call covering hospitalists to address "abnormal" but clinically insignificant findings. Regular monitoring of vital signs is an integral part of caring for acutely ill inpatients but for most inpatients, the importance of vitals is to detect clinically meaningful changes, not to treat risk factors like hypertension that should be treated safely over the long term.

When inpatients become febrile, tachycardic, or hypoxic, hospitalists use critical thinking to diagnose the underlying causes. Unfortunately, high blood pressure is a vital sign that is treated differently. Many hospitalists see it as a number to fix, not a potential sign of a new underlying problem such as uncontrolled pain, anxiety, or medication side effects. ${ }^{8}$ Both groups of authors took the important first step of educating physicians to think critically when called about high blood pressure. Even more importantly, they took steps to change the system and culture in which providers make these decisions in the first place. Future work in this area would be wise to follow in these footsteps, by encouraging collaboration between hospitalist and nurses to create more logical and patient-specific call parameters that could potentially improve nursing-physician communication, and subsequently, patient care.

Changing the culture to limit the use of intravenous antihypertensives will not be easy, but it is necessary. We encourage readers to investigate intravenous antihypertensives in their own hospitals and consider how better communication between nurses and physicians could change their practice. Recalling Mrs. Jones above, the provider should engage her nurse to help confirm that her hypertension is "asymptomatic" and then consider underlying causes such as pain, anxiety, or withholding her home medications as reasons for her elevated blood pressure. After all, if nothing else, it seems clear that a call about inpatient hypertension is not a call to act, but to think.

Disclosures: The authors declare that they have no competing interests.

Funding: Dr. Lucas is supported by the Department of Veterans Affairs, Veterans Health Administration, Office of Research and Development and Dartmouth SYNERGY, National Institutes of Health, National Center for Translational Science (UL1TR001086)

\section{References}

1. Axon RN, Cousineau L, Egan BM. Prevalence and management of hypertension in the inpatient setting: A systematic review. J Hosp Med. 2011;6(7):417422. doi: 10.1002/jhm.804.

2. Global status report on noncommunicable diseases 2010. Geneva, Switzerland: World Health Organization;2011. 3.

3. Herzog E, Frankenberger O, Aziz E, et al. A novel pathway for the management of hypertension for hospitalized patients. Crit Pathw Cardiol. 2007;6(4):150-160. doi: 10.1097/HPC.0b013e318160c3a7

4. Weder $A B$, Erickson S. Treatment of hypertension in the inpatient setting: use of intravenous labetalol and hydralazine. J Clin Hypertens (Greenwich). 2010;12(1):29-33. doi: 10.1111/j.1751-7176.2009.00196.x.

5. Campbell P, Baker WL, Bendel SD, White WB. Intravenous hydralazine for blood pressure management in the hospitalized patient: its use is often unjustified. J Am Soc Hypertens. 2011;5(6):473-477. doi: 10.1016/j. jash.2011.07.002.

6. Gaynor MF, Wright GC, Vondracek S. Retrospective review of the use of as-needed hydralazine and labetalol for the treatment of acute hypertension in hospitalized medicine patients. Ther Adv Cardiovasc Dis. 2017;12(1):7-15. doi: 10.1177/1753944717746613

7. James PA, Oparil S, Carter BL, et al. 2014 evidence-based guideline for the management of high blood pressure in adults: report from the panel members appointed to the eighth Joint National Committee (JNC 8). JAMA. 2014;311(5):507-520. doi: 10.1001/jama.2013.284427.

8. Whelton PK, Carey RM, Aronow WS, et al. 2017 ACC/AHA/AAPA/ABC/ ACPM/AGS/APhA/ASH/ASPC/NMA/PCNA Guideline for the Prevention, Detection, Evaluation, and Management of High Blood Pressure in Adults: A Report of the American College of Cardiology/American Heart Association Task Force on Clinical Practice Guidelines. Hypertension. 2018;71(6):12691324. doi: 10.1161/HYP.0000000000000066.

9. Reducing Unnecessary Treatment of Asymptomatic Elevated Blood Pressure with Intravenous Medications on the General Internal Medicine Wards: A Quality Improvement Initiative. Jacobs ZG, Najafi N, Fang MC, et al. J Hosp Med. 2019;14:XXX-XXX. doi: 10.12788/jhm.3087.

10. Assess Before Rx: Reducing the Overtreatment of Asymptomatic Blood Pressure Elevation in the Inpatient Setting. Pasik SD, Chiu S, Yang J, et al. J Hosp Med. 2019;14:XXX-XXX. doi: 10.12788/jhm.3125

11. Breu AC, Axon RN. Acute treatment of hypertensive urgency. $J$ Hosp Med. 2018;13(12):860-862. doi: 10.12788/jhm.3086. 